\title{
Comparing ultrasound-guided and fiberscope-guided intubation
}

\author{
Shi-Yu Wang ${ }^{1} \cdot$ Fu-Shan Xue ${ }^{2}(1) \cdot$ Ya-Yang Liu $^{2}$
}

Received: 13 October 2017 / Accepted: 20 October 2017 / Published online: 1 November 2017

(C) Japanese Society of Anesthesiologists 2017

To the Editor:

In the recent article by Moustafa et al. [1] in which ultrasound-guided and fiberscope-guided intubation were compared in patients with cervical spine immobilization, the rigid neck collar was removed before intubation and manual inline stabilization of the cervical spine was performed by a provider. Actually, fiberscope-guided intubation does not require removing the rigid neck collar used for cervical spine immobilization. We wish to know whether removal of the collar was necessary because the placement of a rigid neck collar can interfere with ultrasound-guided intubation. If so, it may be a drawback of this method.

Furthermore, the anesthetist performing intubation in group B can be regarded as an experienced operator as he had completed more than 200 fiberscope-guided intubations before this study. The operator in group A was stated to have 7 years of experience in application of ultrasound in the airway and in intensive care, but the readers were not provided with the detailed experience in ultrasound-guided intubation. Thus, it was unclear whether the operator executing the ultrasound-guided intubation was competent in this new airway technique. In fact, experience and competency

This comment refers to the article available at doi:10.1007/ s00540-017-2410-7.

Fu-Shan Xue

Xuefushan@Aliyun.Com

1 Department of Anesthesiology, China-Japan Friendship Hospital, Beijing, China

2 Department of Anesthesiology, Plastic Surgery Hospital, Chinese Academy of Medical Sciences and Peking Union Medical College, 33 Ba-Da-Chu Road, Shi-Jing-Shan District, Beijing 100144, People's Republic of China with any intubation technique are critical for its successful use in clinical practice. In a randomized controlled trial, care is required to ensure that the operators are equally proficient with the studied techniques to avoid bias [2]. We are concerned that the results of this study, such as differences in the first-attempt success rate between groups, may denote different learning curves of operators for the techniques studied, rather than the actual efficiency of these techniques.

\section{Compliance with ethical standards}

Financial statement All authors have no financial support and potential conflicts of interest for this work.

Conflict of interest None declared.

\section{References}

1. Moustafa MA, Arida EA, Zanaty OM, El-Tamboly SF. Endotracheal intubation: ultrasound-guided versus fiberscope in patients with cervical spine immobilization. J Anesth. 2017. doi:10.1007/ s00540-017-2410-7

2. Behringer EC, Kristensen MS. Evidence for benefit vs. novelty in new intubation equipment. Anaesthesia. 2011;66(suppl 2):57-64. 